\title{
Correlation between Resistive Index and Glomerular Filtration Rate in Diabetic and Hypertensive Nephropathy
}

\author{
Khalid Hussain, ${ }^{1}$ Mian Waheed Ahmed, ${ }^{1}$ Munazza Yasmeen, ${ }^{1}$ Usman Khalid Cheema, ${ }^{2}$ Abdul Razaq, ${ }^{3}$ Attiq-ur- \\ Rehman Khan ${ }^{2}$
}

\begin{abstract}
Background: Chronic kidney disease is a morbid condition and its incidence is increasing over a period of time. Various attempts have been done to find a reliable and easily available marker to monitor the progression of the disease. One such indicator is the resistive index which is a noninvasive marker that can be used to look at renal failure. Objective: To validate the relation of the resistive index with glomerular filtration rate so that disease progression can be monitored.

Methodology: This comparative cross sectional study was done at the Department of Urology and Renal Transplantation, DHQ Teaching Hospital Gujranwala, from January 2018 to December 2018. Total 45 patients were enrolled and divided into 3 groups of 15 patients each, 15 patients of diabetic nephropathy, 15 patients of hypertensive nephropathy, and 15 patients were included as the comparison group. GFR and Resistive Index was measured for all patients and correlation was calculated using Pearson test and p-value less than 0.05 taken as significant.

Results: The mean age was 48 years (range 38-65 years) in the diabetic group, 39 years (range 32-58 years) in the hypertensive group, and 30.7 years (22-31 years) in the comparison group. Mean RI was 0.74 in the diabetic group, 0.75 in the hypertensive group, and 0.61 in the control group. High RI was significantly associated with low GFR.

Conclusion: On the renal duplex ultrasonography resistive index can be a helpful predictor for renal progression in patients with moderate renal deterioration due to diabetes and hypertension.

Keywords: Doppler duplex ultrasonography, Resistive index, Diabetes, Hypertension, Chronic kidney disease.

Article Citation: Hussain K, Ahmed MW, Yasmeen M, Cheema UK, Razaq A, Khan AUR. Co-Relation between Resistive Index and Glomerular Filtration Rate in Diabetic and Hypertensive Nephropathy. JSZMC 2021;12(1):07-11. DOI: https://doi.org/10.47883/jszmc.v12i1.41
\end{abstract}

This Open Access article in Journal of Sheikh Zayed Medical College is licensed under a Creative Commons Attribution-NonCommercial 4.0 International License.

\section{Introduction}

Chronic kidney disease (CKD) is an illness of great importance and its prevalence and incidence have been increased many folds in the last decades. ${ }^{1,2}$ Diabetes mellitus and hypertension contribute $40 \%$ and $27 \%$ towards end-stage renal disease respectively. ${ }^{3}$ The kidneys are the prime targets of diabetes and renal failure is the second cause of death after myocardial infarction due to diabetes. Early detection and management of chronic kidney disease hold the promise of decreasing the risk of complications of CKD and preventing kidney failure. One of the challenges, however, is that the early stages of CKD are silent and are detectable only through laboratory analyses. Glomerular filtration rate (GFR) remains the most accurate index of kidney function and is thus key to the early management of the disease. ${ }^{4}$

Management of CKD depends upon an accurate assessment of the levels of kidney function. Many kidney diseases are associated with deterioration of kidney function and with the progression of time many complications like anemia, bone disease, malnutrition, neuropathy, and finally kidney failure. The rate of deterioration of kidney function can be slowed down by routine investigations and keeping strict control of diabetes and hypertension along with taking medications regularly. The risk of progression of chronic kidney disease and renal failure can be slowed down by early management and detection of disease. ${ }^{5}$ Neutrophil gelatinase-associated lipocalin, serum creatinine, and proteins in urine are several biomarkers used to assess renal prognosis in CKD

1. Department of Radiology Gujranwala Medical College and DHQ teaching Hospital. Gujranawala, Pakistan.

Correspondence: Dr. Mian Waheed Ahmed, Department of Radiology Gujranwala Medical College and DHQ Teaching Hospital. Gujranwala, Pakistan.

Received: 15-06-2020

Published: 02-02-2021 
patients but it's very difficult to precisely predict renal outcome. ${ }^{6}$

The renal resistive index (RI) is detected by Doppler ultrasound is the measure of resistance to blood flow in the respective kidney is considered a good indicator of renal vascular resistance. Data from many previous studies shown that renal progression is directly proportional to the high resistive index. ${ }^{7-9}$ However, the data regarding the relationship between renal prognosis and RI values is less in patients with moderate renal dysfunction. Resistive Index (R.I) is defined as the difference between peak systolic velocity minus lowest end-diastolic velocity divided by peak systolic velocity. ${ }^{10}$

The normal range is 0.57 to 0.64 of RI. In a normal adult kidney value of 0.70 is usually regarded as the upper limit. RI can be measured with the help of color Doppler sonography in the main segmental interlobar and arcuate arteries. ${ }^{11}$ Vascular impedance determines the intrarenal hemodynamic changes expressed as R.I obtained from the doppler sonography. ${ }^{12}$

The relation between RI and GFR can be expected on the basis of the fact that decreases in GFR remain permanently decreased if there is permanent renal damage. Vascularity of the kidney is also affected when there is damage of renal parenchyma and this depicts the change in RI. Very little literature is available regarding the co-relation between RI and GFR in the case of diabetic and hypertensive nephropathy. So, this study has been designed to see if there is any correlation between the renal resistive index and glomerular filtration rate in the patient of diabetic (type-2) and hypertensive nephropathy, who are not on maintenance dialysis.

\section{Methodology}

Study design: Comparative Cross Sectional study Study setting: Department of Urology, Dialysis, and Kidney transplantation DHQ Teaching Hospital, Gujranwala. Study duration: From January to December 2018.

Sample size: A total of 45 patients were enrolled including 15 patients of diabetic nephropathy, 15 patients with hypertensive nephropathy and 15 patients were healthy comparison group.

Sampling technique: simple random sampling technique.
Inclusion criteria:

- Patients aged 20 to 80 years

- Patients of both gender

- Patients with diabetic nephropathy

- Patients with hypertensive nephropathy

Exclusion criteria:

- Patients with a single kidney

- $\quad$ Patients undergoing RRT

(Renal Replacement Therapy)

- Patients with renal artery stenosis

- Patients of acute renal failure obstructive uropathy, hemolytic uremic syndrome, and drug-induced nephropathy

Data collection procedure: After approval from the hospital ethical committee patients full filing the inclusion and exclusion criteria were enrolled in the study. Written informed consent was taken.

This study comprised of 3 following groups:

Group A: Group A included 15 cases of nephropathy due to diabetes type-2, not requiring dialysis with average serum creatinine $\leq 5 \mathrm{mg} / \mathrm{dl}$ measured twice. Group B: Group B included 15 cases of nephropathy due to hypertension not requiring dialysis with average serum creatinine $\leq 5 \mathrm{mg} / \mathrm{dl}$ measured twice. Group C: Group C included 15 normal cases who were the live related donors for a kidney transplant. Demographic data including age, gender, duration of diabetes, and hypertension were noted. A general physical examination was done. All the subjects underwent DPTA renal scan to calculate absolute GFR estimation of both kidneys and doppler evaluation was done to calculate the renal resistive index of both kidneys. Patients were examined on ultrasonography in both supine and lateral positions; kidney size and echogenicity were noted. Patients were kept well hydrated; pulse and blood pressure were within normal limits before going for resistive index value measurements and also while doing color doppler sonography excessive transducer pressure was avoided. The resistive index values were determined on main segmental interlobar and arcuate arteries avoiding sinus areas and the average value was calculated. Most of the examinations were performed in the range of 3.5 to $5 \mathrm{MHz}$ on color doppler sonography and it was performed in the lowest possible frequency range because it gives the highest sensitivity to low flow. Data analysis procedure: All data was entered and analyzed using SPSS 22. Quantitative variables were presented as mean and range. Qualitative variables were presented as frequency and percentages. The 
correlation was analyzed between GFR and RI of the comparison group with the help of Pearson correlation using SPSS. A p value less than 0.05 was taken as significant.

\section{Results}

Group A (Diabetic Nephropathy): It included a total of 15 patients, age ranged from 38-65 years with a mean age of 48 years. Duration of history of hypertension before the onset of nephropathy was ranged from 7 to 13 years with a mean duration of hypertension of 9.8 years. The serum creatinine was in the range of $1.7 \mathrm{mg} / \mathrm{dl}$ to $4.2 \mathrm{mg} / \mathrm{dl}$ and the mean value was $3.07 \mathrm{mg} / \mathrm{dl}$. The size of the cortex was in the range of $1.2 \mathrm{~cm}-1.5 \mathrm{~cm}$ and the mean cortex size was $1.3 \mathrm{~cm}$. The resistive index values came to be 0.74 . The mean values of GFR of right and left kidneys were $15.6 \mathrm{ml} / \mathrm{min}$ and $15.7 \mathrm{ml} / \mathrm{min}$ respectively.

Group B (Hypertensive Nephropathy): It included 15 patients with hypertensive nephropathy. The age ranged from 32-58 years with a mean age of 39 years. Duration of history of hypertension before the onset of nephropathy was ranged from 7 to 13 years with a mean duration of hypertension of 9.8 years. The serum creatinine ranged from 2.5$5 \mathrm{mg} / \mathrm{dl}$ with a mean value of $3.6 \mathrm{mg} / \mathrm{dl}$ while the diabetic group showed a mean serum creatinine value of $3.07 \mathrm{mg} / \mathrm{d}$. The cortex size ranged from $0.9 \mathrm{~cm}-1.3 \mathrm{~cm}$ with mean cortex size of $1.1 \mathrm{~cm}$. The mean value of R.I for each unit kidney was 0.75 . The mean value of GFR calculated was $15.34 \mathrm{ml} / \mathrm{minutes}$. The relationship between GFR and RI was analyzed with the help of Pearson correlation using SPSS. Results are shown in table-I, II, III).

Group C (Comparison Group): This group includes 15 cases that were normal subjects. The age ranged from 22 years to 37 years with a mean age of 30.7 years. All the patients in this group were asymptomatic. The size of the cortex ranged from $1.4 \mathrm{~cm}$ to $1.6 \mathrm{~cm}$ the mean value of cortex size was $1.5 \mathrm{~cm}$. The echogenicity of the kidney was normal in the whole of the population of the comparison group. The value of the Resistive Index ranged from 0.58 to 0.63 . The mean value of $\mathrm{RI}$ resulted in 0.61 . The mean values for right and left kidneys were 45.8 and $46.8 \mathrm{ml} / \mathrm{min}$ respectively. The correlation was analyzed between GFR and RI of the comparison group with the help of Pearson correlation using SPSS. A significant correlation was observed between GFR and RI of right kidneys and also between GFR and RI of left kidneys of normal subjects (Comparison Group) $(\mathrm{P}<0.05)$.

Table I: Baseline characteristics of the Study Subjects.

\begin{tabular}{|l|c|c|c|}
\hline Variable & $\begin{array}{c}\text { Diabetes } \\
\text { Mellitus }\end{array}$ & Hypertension & Control \\
\hline $\begin{array}{l}\text { Age (Years) } \\
\text { Range (Mean) }\end{array}$ & $38-65(48)$ & $32-58(39)$ & $22-37(30.7)$ \\
\hline Male & $14(96.7 \%)$ & $10(66 \%)$ & $10(66.6 \%)$ \\
\hline Female & $1(3.3 \%)$ & $5(34 \%)$ & $5(33.4 \%)$ \\
\hline HB (mg/d) & 8.5 & 7.5 & 12.5 \\
\hline Serum Creatinine & 3.07 & 3.6 & 0.9 \\
\hline Echogenicity & $3(20 \%)$ & $3(20 \%)$ & $15(100 \%)$ \\
\hline Normal & $7(46.66 \%)$ & $4(26.66 \%)$ & 0 \\
\hline Grade 1 & $5(33.33 \%)$ & $8(53.33 \%)$ & 0 \\
\hline Grade 2
\end{tabular}

Table II: Mean Values of Resistive Index and GFR.

\begin{tabular}{|l|c|c|c|c|}
\hline \multirow{2}{*}{ Group } & \multicolumn{2}{|c|}{ Right kidney } & \multicolumn{2}{c|}{ Left Kidney } \\
\cline { 2 - 5 } & R.I & GFR & R.I & GFR \\
\hline Diabetes & 0.74 & 15.6 & 0.74 & 15.7 \\
\hline Hypertension & 0.75 & 15.6 & 0.75 & 15.0 \\
\hline Comparison & 0.61 & 45.8 & 0.61 & 46.8 \\
\hline
\end{tabular}

It was astonishing to note that $88(73.9 \%)$ out of 119 were poorly controlled diabetics with $\mathrm{HbA1c}>9 \%$. Similarly, 26 (21.8\%) were having fairly controlled diabetes with $\mathrm{HbA} 1 \mathrm{c}$ between 7.1 to $8.9 \%$. Only 4 (3.4\%) patients had good control of diabetes with HbAlc between 4 to $5.9 \%$ at the first appointment. (Table-II)

Table III: Correlation and p-value between GFR and RI of Diabetic and Hypertensive nephropathy

\begin{tabular}{|c|c|c|c|c|c|}
\hline Group & \multicolumn{2}{|c|}{ Variables } & GFR & RI & P-value \\
\hline \multirow{4}{*}{$\begin{array}{l}\text { Case Group } \\
\text { (Diabetic \& } \\
\text { Hypertensive } \\
\text { nephropathy) }\end{array}$} & \multirow{2}{*}{$\begin{array}{l}\text { Left } \\
\text { Kidney }\end{array}$} & GFR & ---- & 0.378 & \multirow[b]{2}{*}{.039} \\
\hline & & RI & 0.378 & ---- & \\
\hline & \multirow{2}{*}{$\begin{array}{l}\text { Right } \\
\text { Kidney }\end{array}$} & GFR & ---- & 0.206 & \multirow{2}{*}{.274} \\
\hline & & RI & 0.206 & --- & \\
\hline \multirow{4}{*}{$\begin{array}{l}\text { Comparison } \\
\text { Group } \\
\text { (Normal } \\
\text { patients) }\end{array}$} & \multirow{2}{*}{$\begin{array}{l}\text { Left } \\
\text { Kidney }\end{array}$} & GFR & ---- & 0.896 & \multirow{2}{*}{0.000} \\
\hline & & RI & 0.896 & ---- & \\
\hline & \multirow{2}{*}{$\begin{array}{l}\text { Right } \\
\text { Kidney }\end{array}$} & GFR & ---- & 0.772 & \multirow{2}{*}{0.001} \\
\hline & & RI & 0.772 & ---- & \\
\hline
\end{tabular}

\section{Discussion}

This comparative study was done to assess the correlation of GFR and RI so that we can use noninvasive RI as a marker for predicting end stage renal disease. This simple indicator can be calculated 
by using color Doppler, which is readily available in all hospitals. In our study, we compared patients of hypertensive and diabetic nephropathy with normal patients. Both groups of patients were not on renal replacement therapy. The mean RI value is significantly different in diabetic and hypertensive nephropathy patients with serum creatinine $\leq 5 \mathrm{mg} / \mathrm{dl}$ not requiring maintenance hemodialysis as compared to normal subjects. RI values are definitely higher in the subjects with low values of GFR when GFR is higher RI values are low and vice versa. There is an inverse relationship between RI and GFR. RI value is higher in hypertensive patients as compared to the diabetic population. These results are similar to other international studies. . $^{10,11}$

In a study, it was demonstrated that an increase in RI of more than 0.8 shows severe deterioration of renal function. ${ }^{13}$ Another observational study has also shown that RI $>0.7$ is an independent risk factor for CKD progression. ${ }^{14}$ Similar to the present study, patients with higher DM rates were older than those with lower RI in the previous two studies and had lower renal function (e.g., creatinine clearance, $24+/-16 \mathrm{ml} / \mathrm{min} / 1.73 \mathrm{~m} 2 \mathrm{vs}$ $91+/-31 \mathrm{ml} / \mathrm{min} / 1.73 \mathrm{~m} 2) .{ }^{13,14}$ However, patients enrolled had mean RI values $>7.0$, diabetes prevalence $>40 \%$ GFR $<60 \mathrm{ml} / \mathrm{min} / 1.73 \mathrm{~m} 2$ and mean RI values $>7.0$. Kawai et al, ${ }^{8}$ also showed that it could be a useful marker to evaluate and detect the progression of RI in renal disease.

In another study, the RI quality was correlated with renal disease progression. Based on RI values of 0.65 and 0.70 , patients were divided into three groups. There was a big difference in GFR decline between the three groups at 24 months. There was a significant difference between the three classes in the survival rate of declining renal function, according to Kaplan Meier's study. Survival rate was defined as the need for long-term dialysis therapy until the end of a follow-up period of two years. Overt proteinuria ( $>$ or $=1.0 \mathrm{~g} / \mathrm{g}$ creatinine and high RI $(>0.70)$ is considered as a strong predictor of worsening kidney disease according to COX proportional-Hazard Analysis. ${ }^{14}$

Wada et al, ${ }^{14}$ conducted a study with deteriorating GFR on RI reliability. Over the four-year followup, 89 patients among 281 CKD patients presented with worsening renal function. Patients are divided by an RI value of 0.70 into two classes. According to Kaplan-Meier study, in patients with $\mathrm{RI}<0.70$ and $\mathrm{RI}>7.0$ respectively, the event-free levels of declining renal function at 48 months are 0.86 and 0.37 (10g-rank sample, $\mathrm{p}<0.001)$. Overt proteinuria $(>1.0 \mathrm{~g} / \mathrm{g}$ creatinine $)$, low GFR $(<50 \mathrm{ml} /$ $\min / 1.73 \mathrm{~m} 2)$ and high RI $(>0.70)$ have been established as COX proportional-hazard analysis independent predictions of worsening renal function. ${ }^{15}$ All these studies validate the results of our study and prove that RI can be co-related with GFR in monitoring the progression of renal failure in diabetic and hypertensive patients.

\section{Conclusion}

Patients who have a history of hypertension, diabetes mellitus, or serum creatinine above baseline may be followed up with color doppler sonography for measurement of RI value in addition to conventional sonography for measurement of the value of renal length, parenchymal thickness, and cortical echogenicity. RI value $>0.7$ should be followed up frequently. There is a change observed of 0.01 in RI value for a change in GFR of $5-10 \mathrm{ml} / \mathrm{min}$. As the range of RI value is a very narrow very marked change in GFR would reflect a very small change in RI value. RI value definitely declares the severity of the disease.

Authors Contribution: KH: Conception of work, Data Collection and revising. MWA: Conception of work, Design of work, and drafting. MY: Acquisition and analysis of data and drafting. UKC: Conception interpretation of data and revising. AR: Design of work and drafting. ARK: Conception of work and revising.

All authors critically revised and approve its final version.

Conflict of Interest: Author has declared no conflict of interest.

Sources of Funding: The source of funding was self. Disclaimer: None

\section{References}

1. D. S. Keith, G. A. Nichols, C. M. Gullion, J. B. Brown, D. H. Smith. Longitudinal follow-up and outcomes among a population with chronic kidney disease in a large managed care organization. Arch. Intern. Med 2004:164(6): 659-663

2. P. Muntner, J. He, L. Hamm, C. Loria, P. K. Whelton. Renal insufficiency and subsequent death resulting from cardiovascular disease in the United States. J. Am. Soc. Nephrol 2002: 13(3): 745-753

3. R. W. Schrier, Manual of nephrology. Lippincott Williams \& Wilkins, 2014. 
4. G. Manjunath, M. J. Sarnak, A. S. Levey. Estimating the glomerular filtration rate: dos and don'ts for assessing kidney function. Postgrad. Med 2001: 11(6): 55-62

5. I. C. Macdougall, B. Tucker, J. Thompson, C. R. V Tomson, L. R. I. Baker, A. E. G. Raine. A randomized controlled study of iron supplementation in patients treated with erythropoietin. Kidney Int 1996: 50(5): 1694-1699.

6. D. Xiang. Clinical application of neutrophil gelatinase-associated lipocalin in the revised chronic kidney disease classification," Int. J. Clin. Exp. Pathol 2014: 7(10): 7172.

7. S. C. Textor, L. O. Lerman. Paradigm shifts in atherosclerotic renovascular disease: where are we now? J. Am. Soc. Nephrol: 26(9): 2074-2080.

8. T. Kawai. Usefulness of the resistive index in renal Doppler ultrasonography as an indicator of vascular damage in patients with risks of atherosclerosis. Nephrol. Dial. Transplant. 2011:26(10): 3256-3262.

9. N. Bigé. Renal arterial resistive index is associated with severe histological changes and poor renal outcome during chronic kidney disease. $B M C$ Nephrol 2012:13(1): 139.
10. M. Arima. Analysis of the arterial blood flow patterns of normal and allografted kidneys by the directional ultrasonic Doppler technique. J. Uro 1979: 122(5): 587591.

11. J.-H. Chen, Y.-S. Pu, S.-P. Liu, T.-Y. Chiu. Renal hemodynamics in patients with obstructive uropathy evaluated by duplex Doppler sonography. J. Urol.,1993: 150(1): 18-21.

12. L. L. Berland, T. L. Lawson, M. B. Adams, B. L. Melrose, W. D. Foley. Evaluation of renal transplants with pulsed Doppler duplex sonography. J. Ultrasound Med 1982: 1(6): 215-222

13. J. Radermacher, S. Ellis, H. Haller. Renal resistance index and progression of renal disease. Hypertension 2002: 39(2): 699-703.

14. T. Sugiura, A. Wada. Resistive index predicts renal prognosis in chronic kidney disease. Nephrol. Dial. Transplant., 2009: 24(9): 2780-2785.

15. T. Sugiura, A. Wada. Resistive index predicts renal prognosis in chronic kidney disease: results of a 4-year follow-up. Clin. Exp. Nephrol 2011:15(1):114-120. 